\title{
A General and Efficient 2-Amination of Pyridines and Quinolines
}

\author{
Jingjun Yin,* Bangping Xiang,* Mark A. Huffman, Conrad E. Raab, and Ian W. \\ Davies
}

Department of Process Research, Merck Research Laboratories, P. O. Box 2000, Rahway, NJ 07065

jingjun_yin@merck.com, bangping_xiang@merck.com

\section{Supporting Information}

Table of Contents

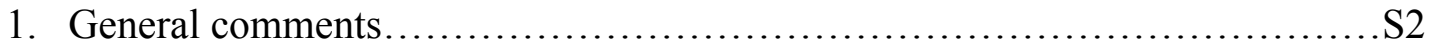

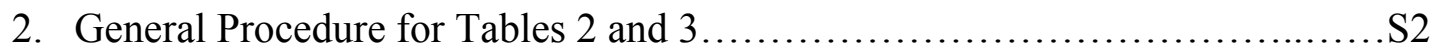

3. Analytic data for 4a,5, 2g,h,k, 11a-n................................S3-S8

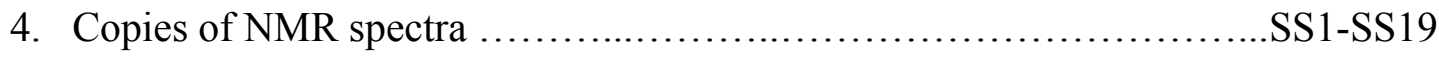




\title{
Supporting Information
}

for

\section{A General and Efficient 2-Amination of Pyridines and Quinolines}

\author{
Jingjun Yin,* Bangping Xiang,* Mark A. Huffman, Conrad E. Raab, and Ian W. \\ Davies \\ Department of Process Research, Merck Research Laboratories, P.O. Box 2000, Rahway, NJ 07065 \\ jingjun_yin@merck.com, bangping_xiang@merck.com
}

General Comments: An HPLC fitted with a C-8 column, auto-injector and diode array detector provided information on reaction progress and assay yield. Relevant HPLC parameters include $0.1 \% \mathrm{H}_{3} \mathrm{PO}_{4}$ in $\mathrm{H}_{2} \mathrm{O} / \mathrm{MeCN}$ mobile phase, $35^{\circ} \mathrm{C}$ column temperature, and monitoring at $210 \mathrm{~nm}$. A $400 \mathrm{MHz}$ NMR system captured all ${ }^{1} \mathrm{H}$, and ${ }^{13} \mathrm{C}$ spectral data associated with all compounds. All columns were packed with 40-63 $\mu \mathrm{m}$ silica gel. Most of starting pyridine $\mathrm{N}$-oxides and (iso)quinoline $\mathrm{N}$-oxides are commercially available.

General procedure of synthesis of 2-amino pyridines and (iso)quinolines: To a RBflask was charged pyridine $N$-oxide or (iso)quinoline $\mathrm{N}$-oxide ( $2 \mathrm{mmol}, 1.0$ equiv.) and solvents as indicated in each entry (mainly trifluorotoluene, dichloromethane or/and chloroform might be added to dissolve the substrates) under $\mathrm{N}_{2}$. After the $\mathrm{N}$-oxide was dissolved, it was cooled to $0{ }^{\circ} \mathrm{C}$ to $10{ }^{\circ} \mathrm{C}$ with an (ice)water bath. t-Butylamine (5.0 equiv) was then added followed by $\mathrm{Ts}_{2} \mathrm{O}\left(2.0\right.$ equiv.) while controlling temp at $5-12{ }^{\circ} \mathrm{C}$ $\left(20{ }^{\circ} \mathrm{C}\right.$ for Table 1 , entry 5$)$. If the reaction was not completed in $30 \mathrm{~min}$, portions of $t$ butylamine ( 0.6 equiv. to 4.0 equiv) and $\mathrm{Ts}_{2} \mathrm{O}$ ( 0.3 equiv. to 2.3 equiv) were charged until 
reaction completed. The $t$-butylaminopyridines could be isolated at this point. However, for the one-pot deprotection, the reaction was treated with TFA $(12 \mathrm{~mL}, 5 \mathrm{~mL}$ for Table 1, entries $1-3 ; 20 \mathrm{~mL}$ for Table 1 , entries $9-10)$ at $70^{\circ} \mathrm{C}$ for $2-6$ hours ( $12 \mathrm{~h}$ for entry 9 ) until $t$-butyl was fully deprotected. Most of the solvents were then removed under vacuum. The residue was diluted with dichloromethane and quenched with $50 \%$ aq $\mathrm{NaOH}$ or sat. sodium carbonate soln to $\mathrm{pH} 9-10$. The aqueous layer was extracted with dichloromethane a few times until no more product was present. The combined organic layers were dried with $\mathrm{Mg}_{2} \mathrm{SO}_{4}$, concentrated and purified by silica gel column chromatography $\left(2-10 \% \mathrm{MeOH} / \mathrm{CH}_{2} \mathrm{Cl}_{2}\right)$ to give the desired 2-aminopyridine or 2-amino (iso)quinoline as a solid. It is also possible to isolate the product as a $\mathrm{HCl}$ salt without column chromatography; see compound $\mathbf{1 1 i}$.

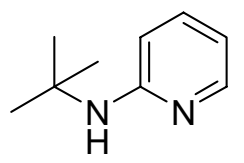

2-tert-butylaminopyridine (4a) ${ }^{1}$ and $\boldsymbol{t}$-butyltosylamide (5): Following the general procedure but stopping before TFA deprotection, pyridine $N$-oxide $(2 \mathrm{mmol}), t$ butylamine (6.0 equiv.) and $\mathrm{Ts}_{2} \mathrm{O}$ (2.5 equiv.) in $10 \mathrm{~mL} \mathrm{PhCF}_{3}$ gave a the reaction mixture of $4 \mathbf{a}$ and 5 . The reaction mixture was quenched with $10 \mathrm{ml}$ water. The organic layer was concentrated and crude ${ }^{1} \mathrm{H}$ NMR $\left(400 \mathrm{MHz}, \mathrm{CDCl}_{3}\right)$ was taken to determine the 2-/4- regioselectivity (4a/10a). Silica gel column chromatography gave $\mathbf{4 a}(92 \%)$ and t-butyltosylamide (5). Data for 4a: ${ }^{1} \mathrm{H} \mathrm{NMR}\left(400 \mathrm{MHz}, \mathrm{CDCl}_{3}\right) \delta 1.43(\mathrm{~s}, 9 \mathrm{H}), 4.50$ (br, $1 \mathrm{H}), 6.40(\mathrm{dt}, J=8.4,1.0 \mathrm{~Hz}, 1 \mathrm{H}), 6.52$ (ddd, $J=7.2,5.2,1.0 \mathrm{~Hz}, 1 \mathrm{H}), 7.34$ (ddd, $J=$ 8.4, 7.2, $2.0 \mathrm{~Hz}, 1 \mathrm{H}), 8.06(\mathrm{ddd}, J=5.2,2.0,1.0 \mathrm{~Hz}, 1 \mathrm{H}) ;{ }^{13} \mathrm{C}\left(100 \mathrm{MHz}, \mathrm{CDCl}_{3}\right) \delta$ $29.6, \quad 50.7,108.7,112.3,136.8,148.1,158.5$. Characteristic data for 4-tertbutylaminopyridine 10a: ${ }^{1} \mathrm{H} \mathrm{NMR}\left(400 \mathrm{MHz}, \mathrm{CDCl}_{3}\right) \delta 8.11(\mathrm{~d}, J=6.4 \mathrm{~Hz}, 2 \mathrm{H})$.

Data for 5: ${ }^{1} \mathrm{H}$ NMR(400 MHz, $\left.\mathrm{CDCl}_{3}\right) \delta 1.21(\mathrm{~s}, 9 \mathrm{H}), 2.41(\mathrm{~s}, 3 \mathrm{H}), 5.01(\mathrm{br}, 1 \mathrm{H}), 7.27$ $(\mathrm{dt}, J=8.4,2.0 \mathrm{~Hz}, 2 \mathrm{H}), 7.79(\mathrm{dt}, J=8.4,2.0 \mathrm{~Hz}, 2 \mathrm{H}) .{ }^{13} \mathrm{C}\left(100 \mathrm{MHz}, \mathrm{CDCl}_{3}\right) \delta 21.5$, $30.2,54.6,127.0,129.5,140.7,142.8$. HRMS calcd for $\mathrm{C}_{11} \mathrm{H}_{17} \mathrm{NO}_{2} \mathrm{SNa}[\mathrm{M}+\mathrm{Na}]^{+}$ 250.0872 , found 250.0866 .

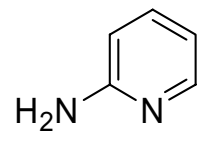

\footnotetext{
${ }^{1}$ Bernardi, P.; Dembech, P.; Fabbri, G.; Ricci, A.; Seconi, G. J. Org. Chem. 1999, 64, 641.
} 
2-Aminopyridine(11a): Following the general procedure, pyridine $N$-oxide ( $2 \mathrm{mmol}$ ), $t$ butylamine (6.0 equiv.) and $\mathrm{Ts}_{2} \mathrm{O}$ (2.5 equiv.) in $10 \mathrm{~mL} \mathrm{PhCF}_{3}$ gave 11a as an off-white solid $(84 \%)$. The spectral data match the commercial material.<smiles>Cc1ccnc(N)c1</smiles>

2-Amino-4-picoline (11b): Following the general procedure, 4-picoline $\mathrm{N}$-oxide (2 mmol), $t$-butylamine (6.6 equiv.) and $\mathrm{Ts}_{2} \mathrm{O}$ (2.8 equiv.) in $10 \mathrm{~mL} \mathrm{PhCF}_{3}$ and $2 \mathrm{~mL}$ $\mathrm{CH}_{2} \mathrm{Cl}_{2}$ gave 11b as an off-white solid (88\%). The spectral data match the commercial material.<smiles>Cc1cccnc1N</smiles><smiles>Cc1ccc(N)nc1</smiles>

2-Amino-3-picoline (11c1) and 2-amino-5-picoline (11c2): Following the general procedure, 3-picoline $\mathrm{N}$-oxide ( $2 \mathrm{mmol}$ ), $t$-butylamine (6.6 equiv.) and $\mathrm{Ts}_{2} \mathrm{O}$ (2.8 equiv.) in $10 \mathrm{~mL} \mathrm{PhCF}$ gave an inseparable mixture of 11c1/11c2 in a 1.7/1 ratio as an off-white solid $(83 \%)$. The spectral data match the commercial material.<smiles>Nc1cc(Cl)ccn1</smiles>

2-Amino-4-chloro pyridine (11d): ${ }^{2}$ Following the general procedure, 4-chloropyridine $\mathrm{N}$-oxide( $2 \mathrm{mmol}$ ), $t$-butylamine(5.0 equiv.) and $\mathrm{Ts}_{2} \mathrm{O}$ (2.0 equiv.) in $10 \mathrm{~mL} \mathrm{CHCl}_{3}$ and 30 $\mathrm{mL} \mathrm{CH}_{2} \mathrm{Cl}_{2}$ gave 11d as a white solid (71\%). ${ }^{1} \mathrm{H} \mathrm{NMR}\left(400 \mathrm{MHz}, \mathrm{CDCl}_{3}\right) \delta 4.65(\mathrm{br}$, $2 \mathrm{H}), 6.54(\mathrm{~d}, J=1.6 \mathrm{~Hz}, 1 \mathrm{H}), 6.67(\mathrm{dd}, J=5.6,1.6 \mathrm{~Hz}, 1 \mathrm{H}), 7.96(\mathrm{~d}, J=5.6 \mathrm{~Hz}, 1 \mathrm{H})$. HRMS calcd for $\mathrm{C}_{5} \mathrm{H}_{6} \mathrm{ClN}_{2}[\mathrm{M}+\mathrm{H}]^{+} 129.02140$, found 129.0217 .

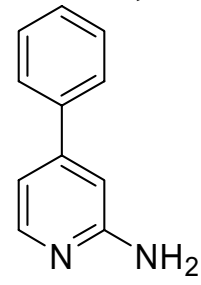

2-Amino-4-phenyl pyridine (11e): ${ }^{3}$ Following the general procedure, 4-phenylpyridine $N$-oxide( 2 mmol), $t$-butylamine(7.5 equiv.) and $\mathrm{Ts}_{2} \mathrm{O}$ (3.8 equiv.) in $10 \mathrm{~mL} \mathrm{PhCF}_{3}$ and 5 $\mathrm{mL} \mathrm{CH}_{2} \mathrm{Cl}_{2}$ gave 11e as an off-white solid (92\%). ${ }^{1} \mathrm{H}$ NMR(400 $\left.\mathrm{MHz}, \mathrm{CDCl}_{3}\right) \delta 4.82$ (br, $2 \mathrm{H}), 6.76(\mathrm{~d}, J=1.4 \mathrm{~Hz}, 1 \mathrm{H}), 6.90-6.92(\mathrm{dd}, J=5.6,1.4 \mathrm{~Hz}, 1 \mathrm{H}), 7.38-7.52(\mathrm{~m}, 3$ $\mathrm{H}), 7.60(\mathrm{dd}, J=6.5,1.7 \mathrm{~Hz}, 2 \mathrm{H}), 8.10(\mathrm{~d}, J=5.6 \mathrm{~Hz}, 1 \mathrm{H}) ;{ }^{13} \mathrm{C}\left(100 \mathrm{MHz}, \mathrm{CDCl}_{3}\right) \delta$ $106.4,112.9,126.5,127.0,128.9,129.0,129.7,138.8,148.3,150.6,158.9$. HRMS calcd for $\mathrm{C}_{11} \mathrm{H}_{11} \mathrm{~N}_{2}[\mathrm{M}+\mathrm{H}]^{+} \quad$ 171.0917, found 171.0920.

\footnotetext{
${ }^{2}$ Meigh, J. P.; Alvarez, M.; Joule, J. A. J. Chem. Soc. Perkin Trans. 1 2001,2012-2021

${ }^{3}$ Wachi, K.; Terada, A. Chem. Pharm. Bull. 1980, 28, 465-472.
} 


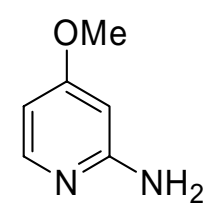

2-Amino-4-methoxy pyridine (11f) ${ }^{2}: \quad$ Following the general procedure, 4methoxypyridine $\mathrm{N}$-oxide hydrate $(2 \mathrm{mmol}), t$-butylamine( 9.0 equiv.) and $\mathrm{Ts}_{2} \mathrm{O}(4.0$ equiv.) in $3 \mathrm{~mL} \mathrm{PhCF}_{3}$ and $7 \mathrm{~mL} \mathrm{CH} \mathrm{Cl}_{2}$ gave 11f as a white solid (90\%). ${ }^{1} \mathrm{H}$ NMR (400 MHz, DMSO-d $\left.{ }_{6}\right) \delta 3.79(\mathrm{~s}, 3 \mathrm{H}), 6.21(\mathrm{dd}, J=2.3,1 \mathrm{H}), 6.33(\mathrm{dd}, J=6.8,2.3 \mathrm{~Hz}, 1 \mathrm{H})$, 7.23-7.32(br m, $2 \mathrm{H}$ ), 7.74-7.84(d, $J=6.8 \mathrm{~Hz}, 1 \mathrm{H})$.

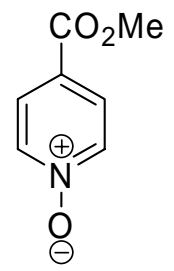

Isonicotinic acid methyl ester $\mathbf{N}$-oxide $(\mathbf{2 g})^{4}$ To a RB-flask $(200 \mathrm{~mL})$ equipped with a magnetic stir-bar and temperature probe was charged isonicotinic acid $\mathrm{N}$-oxide $(2.09 \mathrm{~g}$, $15.0 \mathrm{mmol})$ and methanol $(50 \mathrm{~mL})$. It was cooled to $0{ }^{\circ} \mathrm{C}$ with an ice bath followed by the addition of $\mathrm{TMSCHN}_{2}(2.0 \mathrm{~N}$ in hexane, $22.5 \mathrm{~mL})$. The resulting reaction was aged at 0 ${ }^{\circ} \mathrm{C}$ for $1 \mathrm{~h}$. The solvent was removed and the residue was passed a silica gel pad $\left(5 \% \mathrm{MeOH} / \mathrm{CH}_{2} \mathrm{Cl}_{2}\right)$. The filtrate was concentrated and triturated from AcOEt/Hexane to give the desired product $2 \mathrm{~g}(2.23 \mathrm{~g}, 97 \%)$ as a pale yellow solid. ${ }^{1} \mathrm{H}$ NMR $(400 \mathrm{MHz}$, $\left.\mathrm{CDCl}_{3}\right) \delta 3.93(\mathrm{~s}, 3 \mathrm{H}), 7.85(\mathrm{~d}, J=5.5 \mathrm{~Hz}, 2 \mathrm{H}), 8.21(\mathrm{~d}, J=5.5 \mathrm{~Hz}, 2 \mathrm{H})$.<smiles>CC(=O)c1ccnc(N)c1</smiles>

2-Amino-isonicotinic acid methyl ester (11g): ${ }^{5} \quad$ Following the general procedure, isonicotinic acid methyl ester $N$-oxide ( $2 \mathrm{mmol})$, $t$-butylamine (7.6 equiv.) and $\mathrm{Ts}_{2} \mathrm{O}(3.5$ equiv.) in $10 \mathrm{~mL} \mathrm{PhCF}_{3}$ gave $\mathbf{1 1 g}$ as an off-white solid $(92 \%) .{ }^{1} \mathrm{H} \mathrm{NMR}(400 \mathrm{MHz}$, $\left.\mathrm{CDCl}_{3}\right) \delta 3.92(\mathrm{~s}, 3 \mathrm{H}), 4.62(\mathrm{br}, 2 \mathrm{H}), 7.08(\mathrm{~s}, 1 \mathrm{H}), 7.17(\mathrm{dd}, J=5.2,1.1 \mathrm{~Hz}, 1 \mathrm{H}), 8.20$ $(\mathrm{dd}, J=5.2,1.1 \mathrm{~Hz}, 1 \mathrm{H}) ;{ }^{13} \mathrm{C}\left(100 \mathrm{MHz}, \mathrm{CDCl}_{3}\right) \delta 52.6,108.5,113.0,139.3,148,9$ 159.0, 166.0. HRMS calcd for $\mathrm{C}_{7} \mathrm{H}_{9} \mathrm{O}_{2} \mathrm{~N}_{2}[\mathrm{M}+\mathrm{H}]^{+}$153.0658, found 153.0665 .

\footnotetext{
${ }^{4}$ Copere, C.; Adolfssn, H.; Khuong, T. A. V.; Yudin, A. K. and Sharpless, K. B. J. Org. Chem. 1998, 63, 1740-1741

${ }^{5}$ Kuramochi, T.; Kakefuda, A.; Yamada, H.; Tsukamoto, I.; Taguchi, T.; Sakamoto, S. Bioorg. Med. Chem. 2005, 13, 4022-4036.
} 


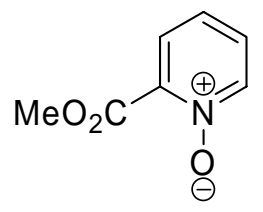

Nicotinic acid methyl ester N-oxide(2h): ${ }^{4}$ Following the procedure of Isonicotinic acid methyl ester $N$-oxide from Nicotinic acid $N$-oxide. The desired product (2h) was obtained (98\% yield) as a brown solid. ${ }^{1} \mathrm{H}$ NMR $\left(400 \mathrm{MHz}, \mathrm{CDCl}_{3}\right) \delta 4.01(\mathrm{~s}, 3 \mathrm{H}), 7.25-7.36(\mathrm{~m}, 2$ H), $7.62(\mathrm{~d}, J=7.7 \mathrm{~Hz}), 8.25(\mathrm{~d}, J=6.4 \mathrm{~Hz}, 1 \mathrm{H})$.<smiles>COC(=O)c1cccc(N)n1</smiles>

2-Amino-nicotinic acid methyl ester $(11 \mathrm{~h}):{ }^{6}$ Following the general procedure, nicotinic acid methyl ester $N$-oxide( $2 \mathrm{mmol}$ ), $t$-butylamine(7.0 equiv.) and $\mathrm{Ts}_{2} \mathrm{O}$ (3.3 equiv.) in 3 $\mathrm{mL} \mathrm{PhCF}_{3}$ and $7 \mathrm{~mL} \mathrm{CH}_{2} \mathrm{Cl}_{2}$ gave 11h as a white solid $(80 \%) .{ }^{1} \mathrm{H}$ NMR $(400 \mathrm{MHz}$, $\left.\mathrm{CDCl}_{3}\right) \delta 3.97(\mathrm{~s}, 3 \mathrm{H}), 4.68(\mathrm{br}, 2 \mathrm{H}), 6.68(\mathrm{dd}, J=8.0,1.0 \mathrm{~Hz}, 1 \mathrm{H}), 7.50-7.58(\mathrm{~m}, 2 \mathrm{H})$<smiles>Nc1cccc(-c2ccccn2)n1</smiles>

2-Amino-4-(2'-pyridyl) pyridine(11i): ${ }^{7}$ Following the general procedure, 4-(2'-pyridyl) pyridine $N$-oxide $(2 \mathrm{mmol}), t$-butylamine (5.3 equiv.) and $\mathrm{Ts}_{2} \mathrm{O}(2.3$ equiv.) in $20 \mathrm{~mL}$ $\mathrm{PhCF}_{3}$ gave 11i as an impure mixture. The mixture was dissolved in MTBE and treated with $\mathrm{HCl}$ (2.0 equiv, $2.0 \mathrm{~N}$ in ether) to give the salt precipitate. The salt was collected by filtration and washed with MTBE. The salt was free-based by portioning in $\mathrm{CH}_{2} \mathrm{Cl}_{2}$ and sat. $\mathrm{NaHCO}_{3}$. The aqueous layer was extracted with $\mathrm{CH}_{2} \mathrm{Cl}_{2}$ several times. The combined organic layers were dried in $\mathrm{MgSO}_{4}$, concentrated and dried under vacuum to give 11i as white solid (81\%). ${ }^{1} \mathrm{H}$ NMR $\left(400 \mathrm{MHz}, \mathrm{CDCl}_{3}\right) \delta 4.52(\mathrm{br}, 2 \mathrm{H}), 6.55(\mathrm{~d}, J=$ $8.0 \mathrm{~Hz}, 1 \mathrm{H}), 7.25-7.28(\mathrm{~m}, 1 \mathrm{H}), 7.58(\mathrm{t}, J=8.0 \mathrm{~Hz}, 1 \mathrm{H}), 7.72-7.80(\mathrm{~m}, 2 \mathrm{H}), 8.27$ (d, $J=$ $8.0 \mathrm{~Hz}, 1 \mathrm{H}), 8.66-8.67(\mathrm{~m}, 1 \mathrm{H}) ;{ }^{13} \mathrm{C}\left(100 \mathrm{MHz} \mathrm{CDCl}_{3}\right) \delta 109.0,111.6,121.0,123.4$, $136.8,138.7,149.2,154.6,156.4,158.1$. HRMS calcd for $\mathrm{C}_{10} \mathrm{H}_{10} \mathrm{~N}_{3}[\mathrm{M}+\mathrm{H}]^{+} 172.0869$, found 172.0864 .<smiles>COc1c(Cl)cc(N)nc1C</smiles>

2-Amino-4-chloro-5-methoxy-6-methyl pyridine(11j): Following the general procedure, 4-chloro-5-methoxy-6-methyl pyridine $N$-oxide $(2 \mathrm{mmol})$, t-butylamine $(9.0$

\footnotetext{
${ }^{6}$ Kelly, T.R.; Lang, F. J. Org. Chem. 1996, 61, 4623-4633.

${ }^{7}$ Mutai, T.; Cheon, J.-D.; Tsuchiya, G.; Araki, K. J. Chem. Soc. Perkin Trans. 2, 2002, 862-865.
} 
equiv.) and $\mathrm{Ts}_{2} \mathrm{O}$ (4.3 equiv.) in $10 \mathrm{~mL} \mathrm{CHCl}_{3}$ gave $11 \mathbf{j}$ as a white solid $(82 \%) .{ }^{1} \mathrm{H}$ NMR $\left(400 \mathrm{MHz}, \mathrm{CDCl}_{3}\right) \delta 2.58(\mathrm{~s}, 3 \mathrm{H}), 3.81(\mathrm{~s}, 3 \mathrm{H}), 7.02(\mathrm{~s}, 1 \mathrm{H}), 7.33(\mathrm{br}, 2 \mathrm{H}) \cdot{ }^{13} \mathrm{C}(100$ $\left.\mathrm{MHz}, \mathrm{CDCl}_{3}\right) \delta 14.4,61.8,111.1,140.8,141.9,147,7,152.2$. HRMS calcd for $\mathrm{C}_{7} \mathrm{H}_{10} \mathrm{ClN}_{2} \mathrm{O}[\mathrm{M}+\mathrm{H}]^{+}$173.0482, found 173.0479 .<smiles>[O-][n+]1cccc2ccccc21</smiles>

Quinoline $N$-oxide (2k): ${ }^{4}$ To a soln of quinoline (98\%) $(5.0 \mathrm{~g}, 37 \mathrm{mmol})$ ) in dichloromethane $(80 \mathrm{~mL})$ at $0{ }^{\circ} \mathrm{C}$ was added $\mathrm{MCPBA}(77 \%)(8.2 \mathrm{~g}, 37 \mathrm{mmol})$. After the reaction was aged at $0{ }^{\circ} \mathrm{C}$ for $1 \mathrm{~h}$, another 1.0 equiv. of MCPBA was added and the reaction was aged at $\mathrm{rt}$ for $3 \mathrm{~h}$. The reaction was filtered. The filtrate was purified by silica gel column (3\% methanol/DCM) to give the pure quinoline $N$-oxide $2 \mathbf{k}(2.70 \mathrm{~g})$ as a pale yellow solid (single run, yield unoptimized) and some impure product contaminated with MCPBA. ${ }^{1} \mathrm{H}$ NMR(400 MHz, CDCl3) $87.31-7.33(\mathrm{t}, J=6.0 \mathrm{~Hz}, 1 \mathrm{H}), 7.64-7.68(\mathrm{t}, J=8.0 \mathrm{~Hz}, 1$ H), 7.75-7.81(m, $2 \mathrm{H}), 7.88-7.90(\mathrm{~d}, J=8.0 \mathrm{~Hz}, 1 \mathrm{H}), 8.56(\mathrm{~d}, J=6.0 \mathrm{~Hz}, 1 \mathrm{H}), 8.77(\mathrm{~d}, J=$ $8.8 \mathrm{~Hz}, 1 \mathrm{H})$.

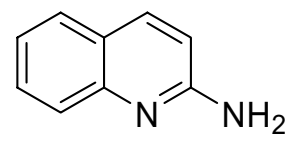

2-Aminoquinoline $(11 \mathbf{k}):{ }^{8}$ Following the general procedure, quinoline $N$-oxide $(2 \mathrm{mmol})$, t-butylamine(5.0 equiv.) and $\mathrm{Ts}_{2} \mathrm{O}$ (2.3 equiv.) in $10 \mathrm{~mL} \mathrm{PhCF}_{3}$ gave $11 \mathbf{k}$ as a white solid (91\%). ${ }^{1} \mathrm{H}$ NMR (400 MHz, $\left.\mathrm{CDCl}_{3}\right) \quad \delta 5.30(\mathrm{br}, 2 \mathrm{H}), 6.71-6.74(\mathrm{dd}, J=8.8,3.5 \mathrm{~Hz}, 1$ H), 7.26-7.30 (m, 1H), 7.57-7.64 (m, 2H), 7.67-7.70 (d, $J=8.3 \mathrm{~Hz}, 1 \mathrm{H}), 7.88-7.90(\mathrm{~d}, J=$ $8.8 \mathrm{~Hz}, 1 \mathrm{H}) ;{ }^{13} \mathrm{C}\left(100 \mathrm{MHz}, \mathrm{CDCl}_{3}\right) \delta 111.9,122.9,123.5,125.4,127.6,130.1,138.5$, 146.9, 156.9. HRMS calcd for $\mathrm{C}_{9} \mathrm{H}_{9} \mathrm{~N}_{2}[\mathrm{M}+\mathrm{H}]^{+}, 145.0766$, found 145.0765 .<smiles>Cc1cc2ccccc2nc1N</smiles>

2-Amino-3-methylquinoline (11l): ${ }^{9}$ Following the general procedure, 3-methylquinoline $N$-oxide( 2 mmol), t-butylamine(5.0 equiv.) and $\mathrm{Ts}_{2} \mathrm{O}$ (2.3 equiv.) in $15 \mathrm{~mL} \mathrm{PhCF}_{3}$ gave $11 \mathrm{l}$ as a white solid $(92 \%) .{ }^{1} \mathrm{H}$ NMR $\left(400 \mathrm{MHz}, \mathrm{CDCl}_{3}\right) \delta 2.31(\mathrm{~s}, 3 \mathrm{H}), 5.0(\mathrm{br}, 2 \mathrm{H})$, $7.25(\mathrm{t}, J=9.0 \mathrm{~Hz}, 1 \mathrm{H}), 7.52(\mathrm{t}, J=8.1 \mathrm{~Hz}, 1 \mathrm{H}), 7.59(\mathrm{~d}, J=8.1 \mathrm{~Hz}, 1 \mathrm{H}), 7.66-7.71(\mathrm{~m}$, $2 \mathrm{H}) ;{ }^{13} \mathrm{C}(100 \mathrm{MHz}, \mathrm{CDCl} 3) \delta 17.6,119.4,122.6,124.6,125.6,126.8,128.8,136.6$, 146.6, 156.7. HRMS calcd for $\mathrm{C}_{10} \mathrm{H}_{11} \mathrm{~N}_{2}[\mathrm{M}+\mathrm{H}]^{+}, 159.0923$, found 159.0917 .

\footnotetext{
${ }^{8}$ Compagnone, R. S.; Suarez, A. I.; Zambran, J. L.; Pina, I. C.; Dominguez, J. N. Synth. Commun. 1997, 27(9), 1631-1641.

${ }^{9}$ Yu, L.; Oost, T. K.; Schkeryantz, J. M.; Yang, J.; Janowick, D.; Fesik, S. W. J. Am. Chem. Soc. 2003, $125,4444-4450$.
} 


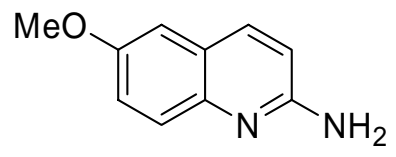

2-Amino-6-methoxy quinoline $(\mathbf{1 1 m}):{ }^{10}$ Following the general procedure, 6-methoxy quinoline $N$-oxide $(2 \mathrm{mmol}), t$-butylamine(5.0 equiv.) and $\mathrm{Ts}_{2} \mathrm{O}(2.3$ equiv.) in $10 \mathrm{~mL}$ $\mathrm{PhCF}_{3}$ and $5 \mathrm{~mL} \mathrm{CH}_{2} \mathrm{Cl}_{2}$ gave $11 \mathrm{~m}$ as a white solid $(83 \%)$. ${ }^{1} \mathrm{H} \mathrm{NMR}\left(400 \mathrm{MHz}, \mathrm{CDCl}_{3}\right)$ $\delta 3.9$ (s, $3 \mathrm{H}), 4.7$ (br, $2 \mathrm{H}), 6.72(\mathrm{~d}, J=8.8 \mathrm{~Hz}, 1 \mathrm{H}), 6.98$ (d, $J=2.5 \mathrm{~Hz}, 1 \mathrm{H}), 7.25$ (dd, $J=9.1,2.5 \mathrm{~Hz}, 1 \mathrm{H}), 7.61(\mathrm{~d}, J=9.1 \mathrm{~Hz}, 1 \mathrm{H}), 7.82(\mathrm{~d}, J=8.8 \mathrm{~Hz}, 1 \mathrm{H}) ;{ }^{13} \mathrm{C}(100$

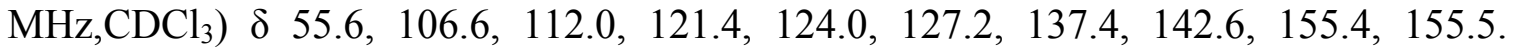
HRMS calcd for $\mathrm{C}_{10} \mathrm{H}_{11} \mathrm{~N}_{2} \mathrm{O}[\mathrm{M}+\mathrm{H}]^{+}, 175.0866$, found 175.0867.

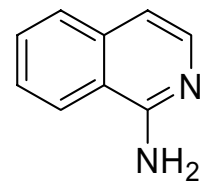

1-Aminoisoquinoline (11n): ${ }^{11}$ Following the general procedure, iosquinoline $\mathrm{N}$-oxide(2 mmol), $t$-butylamine(5.0 equiv.) and $\mathrm{Ts}_{2} \mathrm{O}\left(2.0\right.$ equiv.) in $15 \mathrm{~mL} \mathrm{CHCl}_{3}$ gave $11 \mathrm{n}$ as a white solid (75\%). ${ }^{1} \mathrm{H}$ NMR (400 MHz, $\left.\mathrm{CDCl}_{3}\right) \delta 5.20(\mathrm{br}, 2 \mathrm{H}), 7.07$ (d, $J=5.9 \mathrm{~Hz}, 1$ H), 7.52 (t, $J=6.9 \mathrm{~Hz}, 1 \mathrm{H}), 7.65(\mathrm{t}, J=6.9 \mathrm{~Hz}, 1 \mathrm{H}), 7.72(\mathrm{~d}, J=8.3 \mathrm{~Hz}, 1 \mathrm{H}), 7.82$ (d, $J=8.3 \mathrm{~Hz}, 1 \mathrm{H}), 7.96(\mathrm{~d}, J=5.9 \mathrm{~Hz}, 1 \mathrm{H}) ;{ }^{13} \mathrm{C}\left(100 \mathrm{MHz}, \mathrm{CDCl}_{3}\right) \delta 112.6,117.9,122.8$, 126.4, 127.2, 130.4, 137.4, 140.4, 156.1. HRMS calcd for $\mathrm{C}_{9} \mathrm{H}_{9} \mathrm{~N}_{2}[\mathrm{M}+\mathrm{H}]^{+}, 145.0760$, found 145.0765 .

\footnotetext{
${ }^{10}$ Inglis, S. R.; Stojkoski, C.; Branson, K. M.; Cawthray, J. F.; Fritz, D.; Wiadrowski, E.; Pyke, S. M.; Booker, G. W. J. Med. Chem. 2004, 47, 5405 - 5417.

${ }^{11}$ van Veldhuizen, A.; van Dijk, M.; Sanders, G. M. Org. Magn. Reson. 1980, 13, 105-109.
} 